\title{
Transcatheter Revision of Fontan Circulation by Connecting the Classical Glenn Circulation with the Inferior Vena Cava and Pulmonary Artery Circulation
}

\author{
Vishal R. Kaley, MBBS, MD' ${ }^{1}$, E. Oliver Aregullin, MD, FAAP ${ }^{1,2}$, Stephen C. Cook, MD, FACC ${ }^{1,2}$, \\ Samuel J. Lacina, $M D^{1,2}$, Bennett P. Samuel, MHA, BSN, RN ${ }^{1}$, \\ Joseph Vettukattil, MBBS, MD, DNB, CCST, FRCPCH, FRSM, FRCP ${ }^{1,2^{*}}$ \\ ${ }^{1}$ Congenital Heart Center, Spectrum Health Helen DeVos Children's Hospital, Grand Rapids, Michigan, USA \\ 2 Pediatrics and Human Development, College of Human Medicine, Michigan State University, Grand Rapids, Michigan, USA
}

\begin{abstract}
Patients with single-ventricle physiology require staged palliation. Additional interventions may be required due to Fontan failure, formation of collaterals or pulmonary arteriovenous malformations (PAVMs). Transcatheter interventions are preferable in this setting to avoid the risks of redo-sternotomy, and cardiopulmonary bypass. We present our experience with transcatheter revision of Fontan circulation in a cyanotic adult congenital heart patient with hypoplastic left heart syndrome (HLHS). Transcatheter Fontan completion may be a feasible option in patients with favorable anatomy and hemodynamics with optimal outcomes.

Copyright @ 2019 Science International Corp.
\end{abstract}

\section{Key Words}

Adult congenital heart disease $\cdot$ Hypoplastic left heart syndrome - Pulmonary arteriovenous malformations • Transcatheter intervention

\section{Introduction}

Surgical interventions in patients with single-ventricle physiology are challenging due to the need for staged palliation involving Norwood procedure followed by Glenn and Fontan surgery [1, 2]. Despite ad-

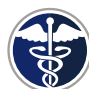

Fax +1 2037853346

E-Mail: jshd@scienceinternational.org

http://structuralheartdisease.org/ (c) 2019 Journal of Structural Heart Disease Published by Science International Corp. ISSN 2326-4004

Accessible online at: http://structuralheartdisease.org/ vancements in surgical technique, these patients are known to develop complications such as formation of collaterals or pulmonary arteriovenous malformations (PAVMs), Fontan failure, and Fontan associated liver disease (FALD) [3-5]. Multiple transcatheter and surgical interventions may be required for treatment of these complications. Transcatheter interventions are preferable in Fontan patients to decrease the need for re-do sternotomy and cardiopulmonary bypass (CPB). We present our experience with transcatheter Fontan completion in a cyanotic adult congenital heart patient with hypoplastic left heart syndrome (HLHS).

\section{Case Presentation}

A 31-year-old woman with HLHS presented with cyanosis and decreasing exercise capacity to an adult congenital heart clinic at a tertiary medical center in the Midwest. She was referred to the congenital heart interventional team to evaluate underlying anatomy, hemodynamics, and source of cyanosis.

After HLHS was diagnosed in the early neonatal period, she underwent stage- 1 palliation. At 6 months of age, she was found to have a widely patent

* Corresponding Author:

Joseph Vettukattil, MBBS, MD, DNB, CCST, FRCPCH, FRSM, FRCP

Congenital Cardiology

Spectrum Health Helen DeVos Children's Hospital

100 Michigan NE (MC48), Grand Rapids, Michigan 49503, USA

Tel. +1 616267 0988; Fax: +1 616267 1408;

E-Mail: joseph.vettukattil@helendevoschildrens.org 

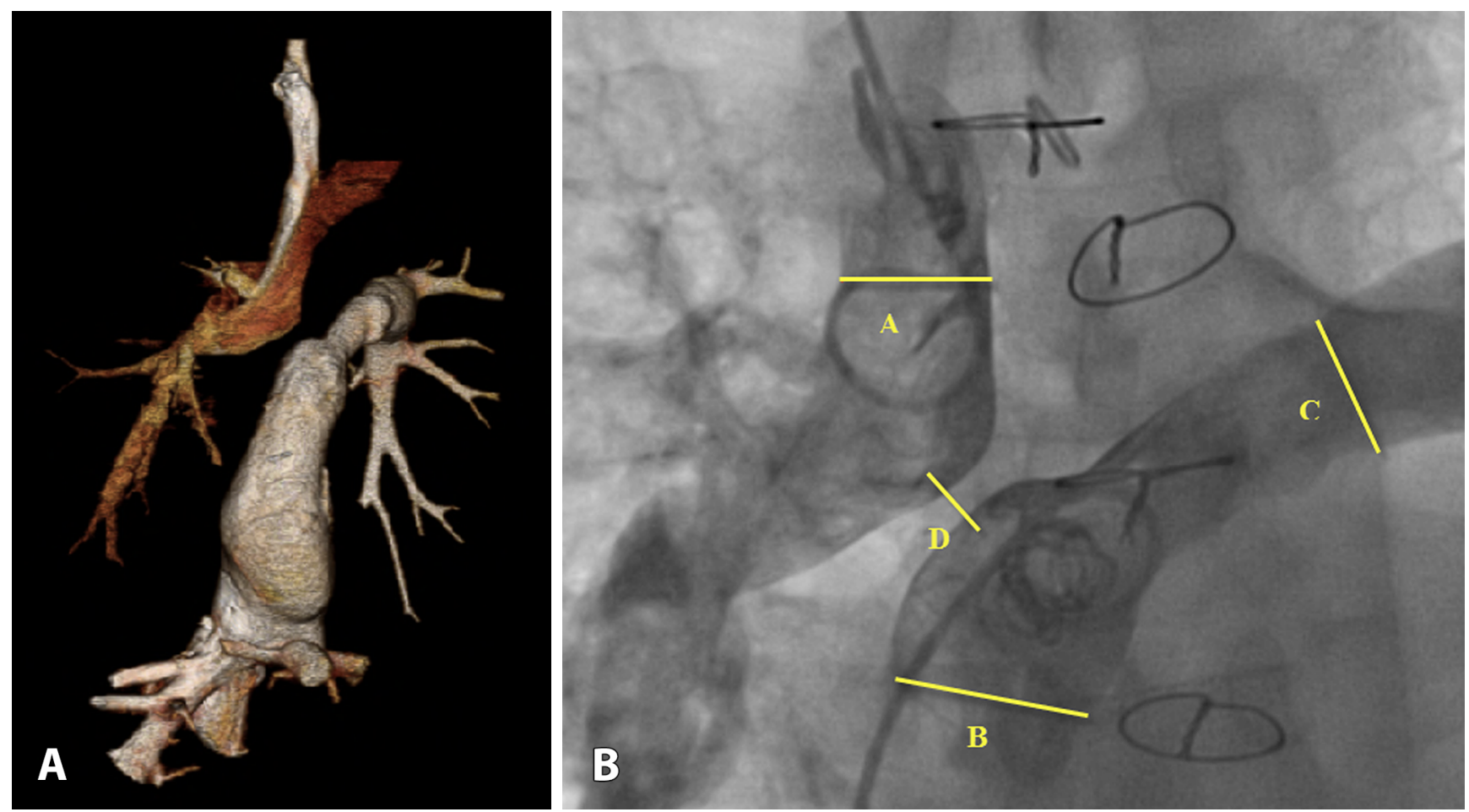

Figure 1. Panel A. Pre-procedure computed tomography scan and Panel B. Pre-procedure angiogram showing the Glenn anastomosis with superior vena cava connected to the right pulmonary artery ( $A=14 \mathrm{~mm}$ ); Fontan conduit (inferior vena cava, $B=18 \mathrm{~mm}$; left pulmonary artery $C=13 \mathrm{~mm}$ ). The distance between the disconnected Glenn and Fontan anastomoses is $D=5 \mathrm{~mm}$.

Gortex aorto-pulmonary shunt with narrowing of the Norwood anastomosis. She then underwent surgical revision of the Norwood anastomosis. A classic-Glenn shunt procedure was also performed connecting the superior vena cava to the right pulmonary artery. At 16 months of age, she underwent Fontan procedure with diversion of the inferior vena cava and hepatic veins to the left pulmonary artery via intra-cardiac baffle. Despite subsequent cardiac catheterizations to occlude multiple veno-venous collaterals, she remained cyanotic.

Prior to cardiac catheterization, a cardiac computed tomography (CT) scan was performed to evaluate underlying anatomy. This demonstrated the anatomy of both the classic Glenn and Fontan anastomoses (Figure 1A). Small PAVMs were noted in the right lower lung lobe along with left-sided veno-venous collaterals. Based on cardiac CT findings, the potential risks associated with re-do sternotomy, and CPB, a multi-disciplinary team recommended transcatheter approach for Fontan completion.
Cardiac catheterization revealed mean Glenn and Fontan pressures of 11 and $15 \mathrm{mmHg}$, respectively. The patient was also noted to have developed hepatic cirrhosis. However, hemodynamic and angiographic assessment reinforced feasibility for transcatheter Fontan completion (Figure 1B). An 8.5 F SL2-transseptal sheath with a 21-gauge transseptal Brockenbrough needle was advanced from the right internal jugular vein into the roof of the Fontan baffle (Figure 2A). Once the needle was placed into the Fontan baffle, a 0.014 inch Mailman wire was advanced and then snared from the femoral vein to create a veno-venous loop. Using a V-18 control wire as a buddy wire, a 0.035 Amplatzer super-stiff wire was placed between the Glenn and Fontan anastomoses. Subsequently, a $28 \mathrm{~mm}$ Cheatham-Platinum (CP) covered stent pre-mounted on a $20 \mathrm{~mm}$ balloon-in-balloon catheter was deployed in between the two circuits (Figure 2B). Post-procedure angiogram demonstrated widely patent Glenn and Fontan anastomoses connected by the covered CP stent with unobstructed flow and favorable hemodynamics (Video 1; Figure 3A). The 

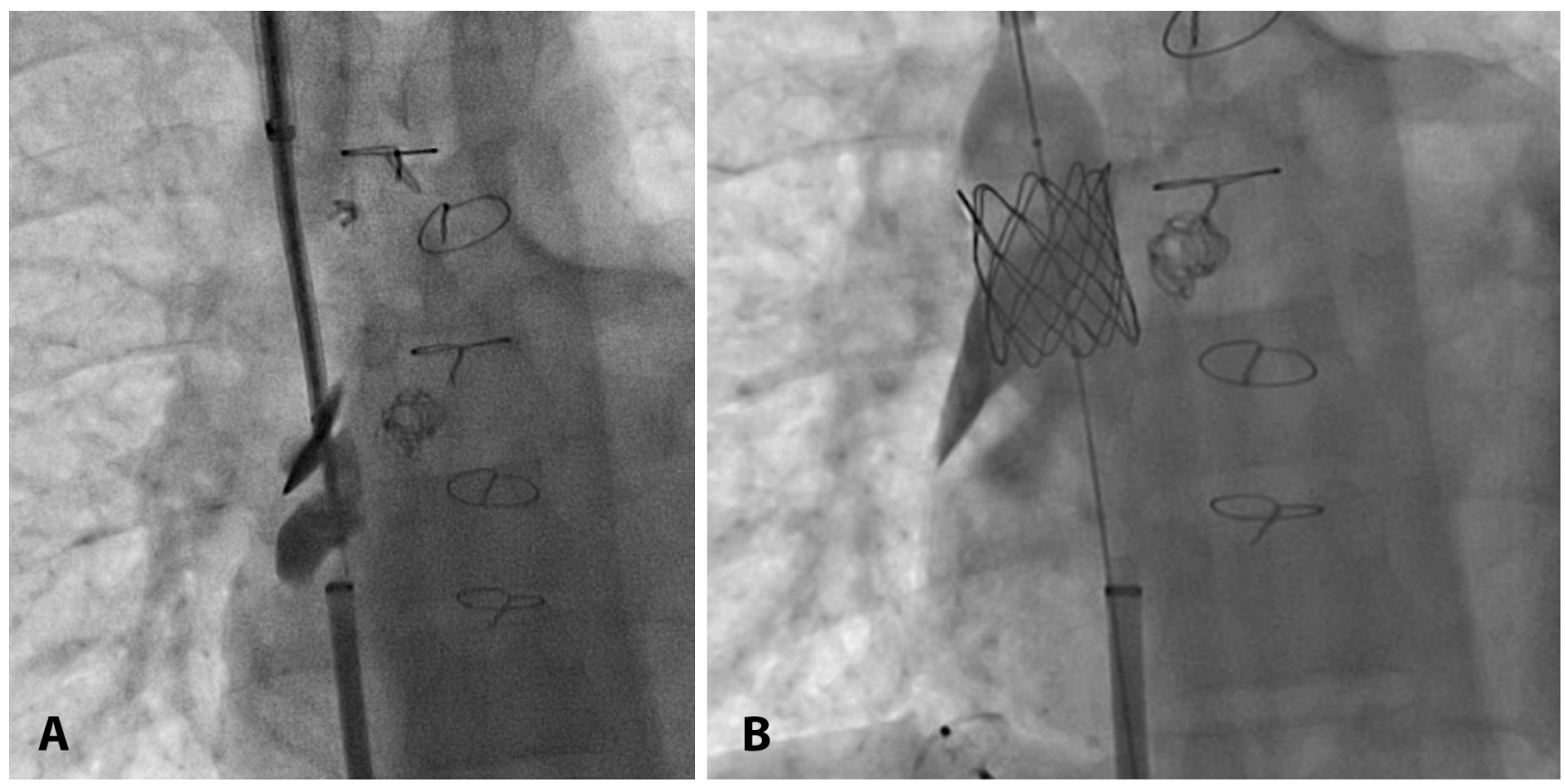

Figure 2. Panel A. Angiogram showing Brockenbrough needle being advanced from the right internal jugular vein into the roof of the Fontan baffle, and Panel B. $28 \mathrm{~mm}$ covered Cheatham Platinum stent placed between the Glenn and Fontan anastomoses.

Fontan pressure decreased to a mean of $13 \mathrm{mmHg}$, while the Glenn pressure was noted to be similar to the pre-procedure mean of $11-12 \mathrm{mmHg}$. The patient was discharged to home the next day. A cardiac CT obtained at 6-week follow-up showed patent Glenn and Fontan anastomoses connected by the covered CP stent (Figure 3B).

\section{Discussion}

The prognosis of patients with single ventricle physiology palliated using the Norwood, Glenn and Fontan procedures is influenced by multiple factors such as the anatomical relationship, flow patterns, and the pressures in the Glenn and Fontan circulations. These patients are at potential risk for the development of complications including PAVMs, Fontan failure, ventricular systolic or diastolic dysfunction, protein losing enteropathy, lymphatic abnormalities, plastic bronchitis, FALD, and reduced glomerular filtration rate $[3,4]$. It is speculated that the risk for developing PAVMs increases when hepatic blood flow carrying hepatic factor; a protective factor to prevent formation of PAVMs, to the lungs is interrupted [5-7]. The risk for developing veno-venous collaterals, Fon-

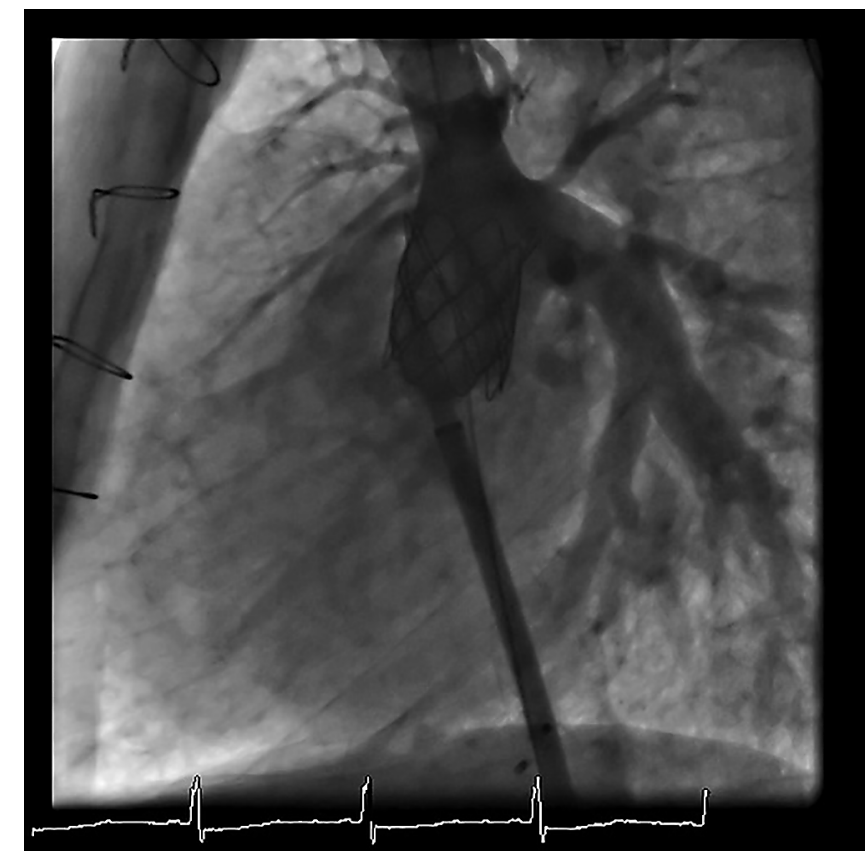

Video 1. Post-procedure angiogram demonstrates widely patent Glenn and Fontan anastomoses connected by the covered CP stent with unobstructed flow and favorable hemodynamics. View supplemental video at https://doi.org/10.12945/j. jshd.2019.017.18.sup.01. 

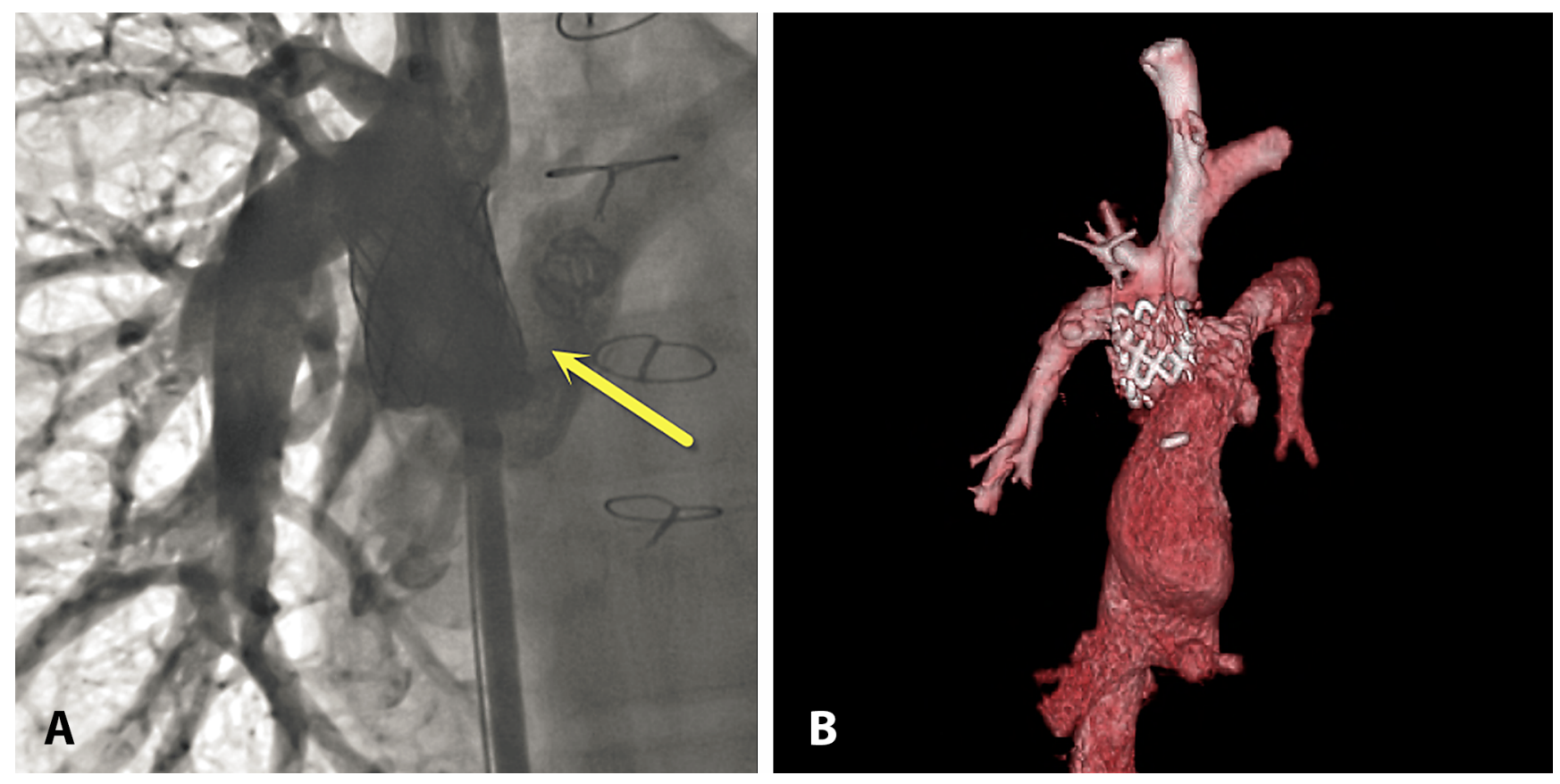

Figure 3. Panel A. Post-procedure angiogram, and Panel B. Follow-up computed tomography scan confirm widely patent Glenn and Fontan anastomoses connected by the $28 \mathrm{~mm}$ CP stent.

tan failure, and FALD is increased in patients with elevated Fontan pressures, which are known to increase further with activity [8]. As a result these patients may require multiple surgical or transcatheter interventions to address these late-onset complications. Surgical interventions are not without risk for these patients given the inherent need for re-do sternotomy, and CPB. As such, transcatheter interventions provide a safer alternative for these patients.

The proof of concept for transcatheter Fontan completion was first demonstrated using specially developed occluding stents in an ovine model [9]. Safety and feasibility in human subjects was then demonstrated in 16 patients with single ventricle physiology with successful transcatheter Fontan completion in 2007 [10]. The technique of transcatheter Fontan completion has been described in which patients underwent a planned modified cavopulmonary anastomosis followed by transcatheter Fontan completion [11]. The technique has not been widely accepted. Patients with traditional Glenn and unidirectional Fontan palliation with PAVMs have benefitted after reconnection of the pulmonary arteries. The feasibility of transcatheter reconnection of the pulmonary arteries in cyanotic patients with PAVMs has been previously described. In this case series of 6 patients with unidirectional Fontan and PAVMs, oxygen saturations were noted to have improved following transcatheter reconnection of the pulmonary arteries. The transcatheter approach was noted to be less invasive with decreased morbidity compared to surgical pulmonary artery reconnection or brachial arteriovenous fistula placement [12].

The unique condition of developing PAVMs in the right lung and veno-venous collaterals in left lung in our patient may be explained by the absence of hepatic flow into the right lung and exposure of the left lung to higher Fontan pressures [6]. The liver cirrhosis may also be attributed to high pressures in the Fontan circuit. Anticipating the potential benefits of connecting both the Glenn and Fontan anastomoses to achieve even distribution of blood into the lungs, transcatheter approach for Fontan completion was selected as an alternative approach to surgical intervention.

Transcatheter Fontan completion was performed without preconditioning in this patient due to functional decline associated with chronic cyanosis, to avoid re-do sternotomy and CPB and multi-organ complications associated with Fontan failure and 
chronic cyanosis. The procedure was successful with immediate fall in the Fontan pressures and optimal flow and equal distribution of blood to the lungs.

As such, transcatheter Fontan completion may be a feasible option in selected adult congenital patients with optimal outcomes even without surgical preconditioning. However, further studies in a larger cohort of are required to evaluate long-term outcomes.

\section{References}

1. Norwood WI, Lang P, Hansen DD. Physiologic repair of aortic atresia-hypoplastic left heart syndrome. N Engl J Med 1983;308:23-26. PMID: 6847920

2. Gordon BM, Rodriguez S, Lee M, Chang RK. Decreasing number of deaths of infants with hypoplastic left heart syndrome. J Pediatr 2008;153:354-358. DOI: 10.1016/j. jpeds.2008.03.009

3. Baek JS, Bae EJ, Ko JS, Kim GB, Kwon BS, Lee SY, et al. Late hepatic complications after Fontan operation; non-invasive markers of hepatic fibrosis and risk factors. Heart 2010;96:1750-1755. DOI: 10.1136/ hrt.2010.201772

4. Book WM, Gerardin J, Saraf A, Marie Valente A, Rodriguez F 3rd. Clinical Phenotypes of Fontan Failure: Implications for Management. Congenit Heart Dis. 2016;11:296308. DOI: $10.1111 /$ chd. 12368

5. Duncan BW, Kneebone JM, Chi EY, Hraska V, Isik FF, Rosenthal GL, et al. A detailed histologic analysis of pulmonary arteriovenous malformations in children with cyanotic congenital heart disease. JThorac Cardiovasc Surg 1999;117:931-938. PMID: 10220688
6. Vettukattil JJ. Is the hepatic factor a miRNA that maintains the integrity of pulmonary microvasculature by inhibiting the vascular endothelial growth factor? Curr Cardiol Rev 2017;13:244-250. DOI: 10.2174/15734 03X13666170510115628

7. Vettukattil JJ, Slavik Z, Lamb RK, Monro JL, Keeton BR, Tsang VT, et al. Intrapulmonary arteriovenous shunting may be a universal phenomenon in patients with the superior cavopulmonary anastomosis: A radionuclide study. Heart 2000;83:425-428. PMID: 10722543

8. Navaratnam $D$, Fitzsimmons $S$, Grocott $M$, Rossiter HB, Emmanuel Y, Diller GP, et al. Exercise-induced systemic venous hypertension in the Fontan circulation. Am J Cardiol 2016;117:1667-1671. DOI: 10.1016/j. amjcard.2016.02.042

9. Metton O, Calvaruso D, Stos B, Ben Ali W, Boudjemline $Y$. A new surgical technique for transcatheter Fontan completion. Eur J Cardiothorac Surg 2011;39:81-85. DOI: 10.1016/j.ejcts.2010.04.023

10. Sallehuddin A, Mesned A, Barakati M, Fayyadh MA, Fadley F, Al-Halees Z. Fontan completion without surgery. Eur J
Cardiothorac Surg 2007;32:195-200. DOI: 10.1016/j.ejcts.2007.03.039

11. Hausdorf G, Schneider M, Konertz W. Surgical preconditioning and completion of total cavopulmonary connection by interventional cardiac catheterisation: a new concept. Heart. 1996;75:403-409. PMID: 8705770

12. AboulHosn J, Danon S, Levi D, Castellon Y, Child J, Moore J. Regression of pulmonary arteriovenous malformations after transcatheter reconnection of the pulmonary arteries in patients with unidirectional Fontan. Congenit Heart Dis. 2007;2:179-184. DOI: 10.1111/j.1747-0803.2007.00094.x

Cite this article as: Kaley VR, Aregullin EO, Cook SC, Lacina SJ, Samuel BP, Vettukattil J.Transcatheter Revision of Fontan Circulation by Connecting the Classical Glenn Circulation With the Inferior Vena Cava and Pulmonary Artery Circulation. Structural Heart Disease. 2019;5(1):1115. DOI: https://doi.org/10.12945/j. jshd.2019.017.18 